\title{
Insight into the Stunting vis a vis Salmonella and Shigella Species among Children of Pakistan
}

\section{Batool M', Noreen Z', Krishin $\mathrm{J}^{2}$ and Bokhari $\mathrm{H}^{1 *}$ ${ }^{1}$ Department of Biosciences, COMSATS University, Islamabad 44000, Pakistan \\ ${ }^{2}$ Department of Pediatrics, Pakistan Institute of Medical Sciences (PIMS) Islamabad 44000, Pakistan}

*Corresponding author: Habib Bokhari, Department of Biosciences, COMSATS University Islamabad, 44000, Islamabad, Pakistan

Received: April 15, 2021; Accepted: May 15, 2021;

Published: May 22, 2021

\begin{abstract}
Pakistan is one of the leading countries where the high childhood mortality (under the age of 5 years) as well as is a country where $>33 \%$ of children are underweight and $38 \%$ show stunted growth. The current study investigated the presence or absence of Salmonella and Shigella $s p$. in stunted children under five years of age from the lower socioeconomic background of Pakistan. Besides, the antibiotics susceptibility patterns were studied along with the sociodemographic and clinical information demographic factors using a questionnaire. The stool samples from stunted children have processed following standard bacteriological protocols and presumptive colonies of Salmonella and Shigella species were identified and sub-cultured on selective media and confirmed by using the standard biochemical test as well as molecular tests. Antibiotics susceptibility of the isolates to 10 antibiotics was tested using disk diffusion assay. The results suggested that $10.5 \%$ and $5.7 \%$ of the stool samples were positive for Salmonella and Shigella sp. respectively. Moreover, the antibiotics susceptibility test results of the isolates showed that Salmonella $s p$., were showing higher resistance to amoxicillin whereas Shigella $s p$. were more resistant to gentamycin. All Salmonella and Shigella isolates were resistant to Rifampicin and $80 \%$ of isolates of both were susceptible to ciprofloxacin and cefotaxime. The study suggested that environmental enteric dysfunction (EED), is widespread among malnourished children and may result in stunted growth. The contributory factors such as unsafe farming practices or close association to poultry or livestock animals and prevailing sanitation \& hygiene conditions are the potential source of entero-pathogens.
\end{abstract}

Keywords: Salmonella sp.; Shigella sp.; Antibiotic susceptibility; Stunted growth; Gut diarrheagenic pathogens

\section{Abbreviations}

WHO: World Health Organization; SD: Standard Deviations; Height-for-Age: HAZ; XLD agar: Xylose Lysine; DA: Deoxycholate Agar; SS agar: Salmonella Shigella agar; EED: Environmental Enteric Dysfunction; PCR: Polymerase Chain Reaction; IEVRP: International Enteric Vaccines Research Program; AOR: Adjusted Odds Ratio; CI: Confidence Interval; PCM: Protein-calorie Malnutrition

\section{Introduction}

Almost 3.5 million children die annually, due to maternal and childhood undernutrition [1]. Malnutrition encompasses both overnutrition (overweight \& obesity) and under-nutrition (underweight, stunting \& wasting). Moreover, it is the key problem with detrimental impacts on normal survival, development and economic productivity of individuals and societies at large [2]. Nearly 1.1 billion populations from developing countries have no access to safe drinking water, consequently, they develop enteric infections and besides, due to extreme poverty, most vulnerable children population, in particular, suffer from undernutrition/malnutrition and malabsorption of nutrients and low immunity levels which lead to enhanced susceptibility to infections. This malicious cycle of stunting starts from in utero during the pregnancy and it prevails for several generations resulting in low Body Mass Index (BMI) and IQ of a newly born and continues in early childhood [3]. Various studies have found a correlation between diarrheal pathogens and malnutrition in children with a cumulative 50 per cent of annual deaths of children under five years of age [4]. The most prevalent form of undernutrition is stunting; which results from failure to receive adequate nutrition over an extended period [6].

According to World Health Organization (WHO), wasting, underweight and stunting can be defined as Z-scores more than -2 (>2) standard deviation of weight for height, weight for age and height for age respectively [7]. Moreover, children with height for age below Three Standard Deviations (-3SD), considered severely stunted, from the median of the reference population [8]. According to World Health Organization (WHO), stunting or lower linear growth have several short as well as long term consequences. The short term consequences of stunting among children are morbidity and mortality due to infections such as pneumonia and diarrhoea. Moreover, stunting can significantly affect the development of the brain in the fetus or newborn, poor cognitive and educational outcomes in later childhood as well as in adolescence [9]. Pakistan is among the countries, with the highest rates of stunting globally [10]. According to the Demographic and Public Health survey of Pakistan, conducted in 2019, 38 per cent of Pakistani children who are under five have stunted growth [11]. The determinants of childhood undernutrition can be grouped into; primary factors and secondary factors. The primary factors are the inherent factors (child's age and gender), 


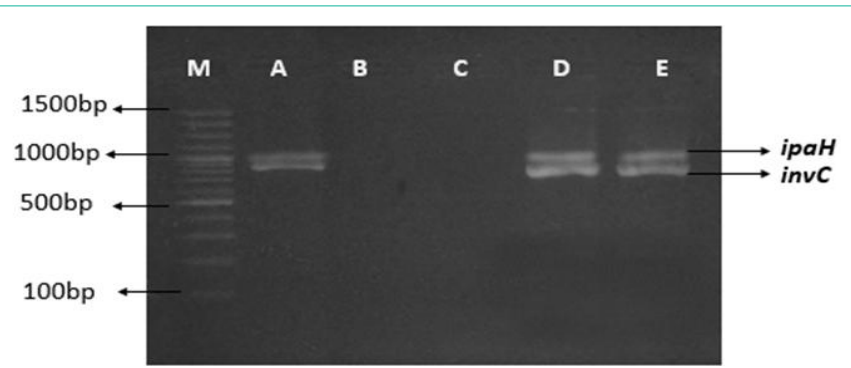

Figure 1: 2\% Agarose gel image showing the combination of Shigella genus isolated from stunted growth children. First lane $\mathrm{M}$ is $100 \mathrm{bp}$ ladder, while $A$ (G069 strain), D (G088 strain), E (G092 strain) shows the Shigella genus. B and $\mathrm{C}$ are negative control for invC and ipaH genes of Shigella sp.

whereas the secondary factors are distal factors which include the socioeconomic status of children, next are the intermediate factors i.e. environmental and maternal factors and the proximate factors such as child feeding, personal hygiene and health status [12]. Children from underprivileged backgrounds have a higher burden of mortality from diarrhoea in developing countries, whereas the annual mortality rate is 1.5-5.1 million [13]. Pakistan is a large livestock raising country [14] and poultry farming has become a common household business and women from the low-income group in rural and urban areas are heavily involved in domestic farming and hence the risk of frequent exposure of vulnerable children population at a very early age to enteropathogens including E. coli, Campylobacter sp., and Shigella sp. which correlate with stunted growth and subsequent life-long physical and cognitive impairments The abundance of such pathogenic bacteria of animal origin interferes with the normal functioning of the gut or small intestine [15], which inhibits the absorption of nutrients, consequently leading to either frequent episodes of diarrhoea while long term asymptomatic association/carriage may lead to stunting [16]. Moreover, the presence of antibiotics resistance among these enteropathogens affects the recovery process. However, their asymptomatic association with the gut of a child with stunted growth has not been systematically investigated and the present study has investigated the occurrence or persistence of Shigella sp. and Salmonella sp. in stunted Pakistani children [17] (Figure 1).

The present study has investigated the positive correlation between gut diarrheagenic pathogens i.e. Shigella sp. and Salmonella $s p$. and childhood stunting in Pakistani children. Moreover, enhanced resistance to commonly used antibiotics to Shigella sp. and Salmonella $s p$. has become a global public health concern in particular when it is associated with such vulnerable children population. Therefore, this study aimed at determining the prevalence and antimicrobial resistance patterns of Salmonella and Shigella sp. among stunted children of Pakistan, under five years of age.

\section{Materials and Methods}

\section{Sampling}

The stool samples were collected from 105 children, under five years of age, who have confirmed stunted growth, based upon the World Health Organization (WHO) formula of height-for-age (HAZ). The children were at more than -2 standard deviation $(>-2)$ from normal growth. Total 20 samples were collected from Swat KPK, 12 from Thar Sindh, 32 from Mayo Hospital Lahore and 41 from PIMS
Hospital Islamabad Pakistan between November 2018 to November 2019. All samples were collected in sterilized tubes containing CarryBlair media. Immediately after collection, samples were shipped on ice to the microbiology laboratory of COMSATS University Islamabad. Moreover, consent forms were filled by the parents or caretakers of children along with the detailed questionnaires that contain particulars of children and families, including dietary intake, water source, health status, sanitary condition and household possession of poultry-livestock animal etc.

\section{Isolation and biochemical identification of Salmonella and Shigella sp.}

The stool samples were inoculated in selective sterilized media i.e., Xylose Lysine Deoxycholate (XLD) agar and Salmonella Shigella (SS) agar plates and were at an incubator for $18-24 \mathrm{hrs}$ at $37^{\circ}$ Presumptive colonies were based on growth and morphology of colonies were subjected to biochemical tests i.e., Motility test, Gram Staining, Urease, Citrate, Oxidase and Catalase. After the confirmation of Salmonella and Shigella sp. isolates were further confirmed through molecular analysis.

\section{Molecular identification of Salmonella and Shigella sp.}

Genomic DNA was extracted from all isolates of Shigella sp. and Salmonella sp., by Ethanol precipitation method [20]. Molecular identification of Shigella $s p$. isolated from stool samples of stunted children was done by Polymerase Chain Reaction (PCR) using specific primers and probes. Two sets of primers targeting invC gene (F 5'TGC CCA GTT TCT TCA TAC GC 3' and R 5'GAA AGT AGC TCC CGA AAT GC 3') and ipaH (F 5'-CTCGGCACGTTTTAATAGTCTGG-3' and R 5'-GTGGAGAGCTGAAGTTTCTCTGC-3') with a product size of $875 \mathrm{bp}$ and $933 \mathrm{bp}$ respectively were used for the detection of Shigella sp. The primer sequences presented in Table 1. The final volume of PCR was $25 \mu \mathrm{l}[21,22]$. A plasmid containing the target $i p a H$ gene i.e., pOG392 (as a precious gift from Oscar G. GomezDuarte, International Enteric Vaccines Research Program [IEVRP], University of Iowa Children's Hospital, Iowa City, IA) was used as the positive control. The molecular analysis of Salmonella sp. isolates from stool samples of stunted children was carried by PCR using specific primers and probes. A set of primers (invA), forward 5'AAA CGT TGA AAA ACT GAG GA 3' and reverse 5'TCG TCA TTC CAT TAC CTA CC 3' with a product size of $119 \mathrm{bp}$ were used for the detection of Salmonella sp. The primer sequences presented in Table 1 and the final volume of PCR was $25 \mu \mathrm{l}$ [23].

\section{Antibiotic susceptibility}

The antibiotics susceptibility of the isolates was tested using disc diffusion method using Rifampicin $(5 \mu \mathrm{g})$, Cefotaxime $(30 \mu \mathrm{g})$,

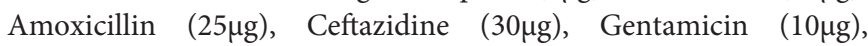

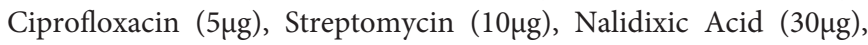
Norfloxacin $(10 \mu \mathrm{g})$ and Ampicillin $(10 \mu \mathrm{g})$. The zone of inhibition was recorded after 24 hours of incubation and the results were interpreted using Clinical Laboratory Standard International Guidelines (2016).

\section{Statistical analysis}

The data were edited and analyzed using SPSS version 25. Multivariate logistic regression test, Adjusted Odds Ratio (AOR), and $95 \% \mathrm{CI}(P<0.05$ significance level) were used to assess the level of association among prevalence of the pathogens and associated risk 


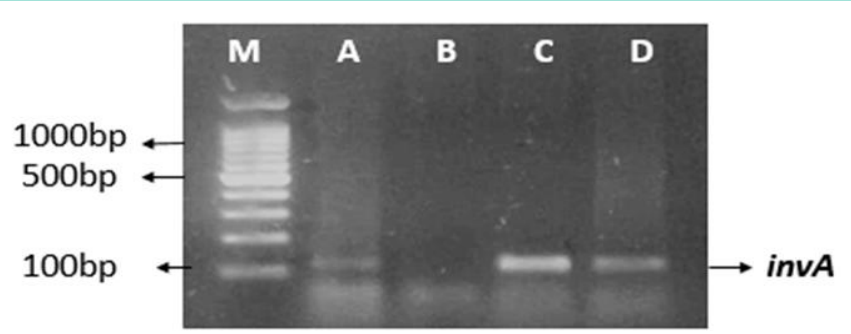

Figure 2: $2 \%$ Agarose gel image showing the combination of Salmonella genus isolated from stunted growth children. First lane $M$ is 100 bp ladder, while A (S025 strain), C (S062 strain), D (S0100 strain), shows Salmonella $s p$. B is the negative control for the invA gene of Salmonella $s p$.

Table 1: List of primer sequence used for Shigella sp. and Salmonella sp.

\begin{tabular}{|c|c|c|c|c|}
\hline $\begin{array}{l}\text { Target } \\
\text { Gene }\end{array}$ & Primer & Primer Sequence & $\begin{array}{l}\text { Product } \\
\text { size }\end{array}$ & Ref. \\
\hline \multirow{2}{*}{ invC } & SgenDF & TGC CCA GTT TCT TCA TAC GC & \multirow{2}{*}{$875 b p$} & \multirow{2}{*}{ [22] } \\
\hline & SgenDR & GAA AGT AGC TCC CGA AAT GC & & \\
\hline \multirow{2}{*}{ invA } & invAF & AAA CGT TGA AAA ACT GAG GA & \multirow{2}{*}{$119 b p$} & \multirow{2}{*}{ [23] } \\
\hline & invAR & TCG TCA TTC CAT TAC CTA CC & & \\
\hline іран & $\begin{array}{l}\text { ipaHF } \\
\text { ipaHR }\end{array}$ & $\begin{array}{l}\text { 5'-CTCGGCACGTTTTAATAGTCTGG-3' } \\
\text { 5'-GTGGAGAGCTGAAGTTTCTCTGC-3' }\end{array}$ & 933bp & [21] \\
\hline
\end{tabular}

factors.

\section{Results}

\section{Prevalence of stunting in different age groups}

It has been recorded that the prevalence of stunting in Pakistan is higher among children below 1 year of age e.g. out of the total of 105 samples collected in this study, 46.6 per cent of children were below 1 year of age and the remaining 53.4 per cent were between 1 to 5 years of age. Table 2 shows that the prevalence of stunting among the 4-5year age group is lowest i.e. $7.9 \%$, whereas $14 \%$ in the $3-4$ year, $9.5 \%$ in the 2-3-year age group (Figure 2).

\section{Sociodemographic and clinical characteristics of the patients}

The majority $(91.1 \%)$ of the partakers were urban dwellers. Moreover, most (55\%) of the children's source of drinking water was tap, whereas $13 \%$ of them consumed boiled water (Table 2).

\section{Prevalence of Salmonella sp. and Shigella sp.}

Molecular analysis was performed by PCR for the detection of the Shigella genus. The incidence ratio for Shigella sp. was $5.7 \%$ among the samples collected from stunted children. $50 \%$ of the Shigella sp. isolates were obtained from female children, whereas, $83 \%$ of the isolates were obtained from children with age less than 24 months. Our study has found no significant correlation $(\mathrm{P}<0.05)$ between gender $(\mathrm{P}<0.57)$, age group $(\mathrm{P}<0.943)$, source of drinking water $(\mathrm{P}<0.820)$, area of residence $(\mathrm{P}<0.351)$ of studied children and prevalence of Shigella sp.

The presence of Salmonella sp. was confirmed through molecular analysis or PCR. The incidence ratio for Salmonella sp. was $10.5 \%$ among the samples collected from stunted children. Almost 54.5\% of the Salmonella $s p$. isolates were obtained from female children. Moreover, this study has found no significant correlation $(\mathrm{P}<0.05)$ between gender $(\mathrm{P}<0.621)$, age group $(\mathrm{P}<0.733)$, source of drinking water $(\mathrm{P}<0.998)$, of the participants and prevalence of Salmonella $s p$. Whereas, a significant correlation has been found between urban dwellers $(\mathrm{P}<0.097)$ and the prevalence of Salmonella sp.
Table 2: Sociodemographic characteristics and prevalence of Salmonella and Shigella sp. among stunted children attending Mayo and PIMS Hospital, February 2019 to February 2020.

\begin{tabular}{|l|c|c|}
\hline \multicolumn{1}{|c|}{ Variables } & Frequency & \% \\
\hline Gender & & \\
\hline M & 49 & $46.67 \%$ \\
\hline F & 56 & $53.33 \%$ \\
\hline Age & & \\
\hline 0-1y & 49 & $46.66 \%$ \\
\hline 1y-2y & 33 & $31.42 \%$ \\
\hline 2y-3y & 10 & $9.50 \%$ \\
\hline 3y-4y & 15 & $14.28 \%$ \\
\hline 4y-5y & 8 & $7.60 \%$ \\
\hline Source of Drinking Water & & \\
\hline Filtered & 33 & $31.00 \%$ \\
\hline Boiled & 14 & $13.00 \%$ \\
\hline Well water & 58 & $55.00 \%$ \\
\hline Residence Area & & 11 \\
\hline Urban & $96.50 \%$ \\
\hline Rural & 9 & $91.00 \%$ \\
\hline Prevalence of Salmonella \& Shigella sp. & 13 & $8.60 \%$ \\
\hline Salmonella sp. & & \\
\hline Shigella sp. & & $12.40 \%$ \\
\hline Total prevalence & & \\
\hline & & \\
\hline
\end{tabular}

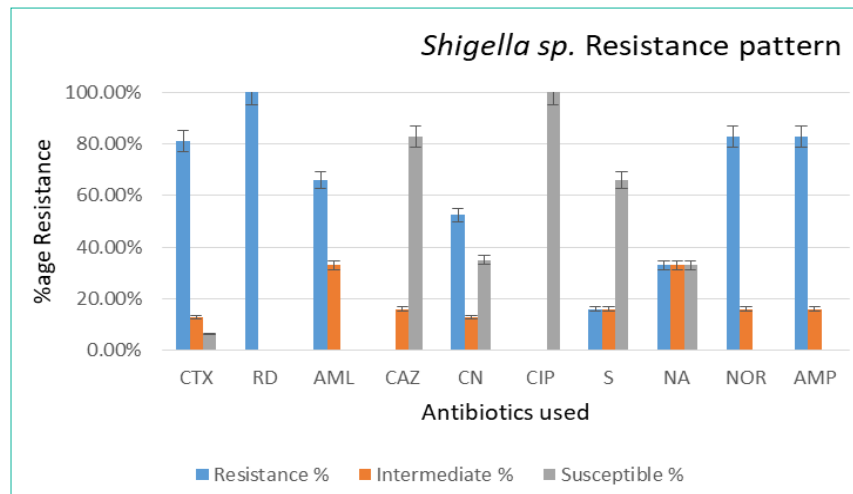

Figure 3: Chart presents resistance pattern of isolated Shigella sp. strains against antibiotics used.

Interestingly, this study has found a significant association $(\mathrm{P}<0.05)$ between the prevalence of Salmonella sp. in stunted children with isolation of Shigella sp (AOR: 6.4, $\mathrm{P}<0.001$ ) from them. However, a large study cohort may give a more clear picture.

\section{Antibiotic susceptibility pattern of the isolates}

Of all Shigella sp. isolates, $100 \%$ were resistant to Rifampicin $(5 \mu \mathrm{g})$, whereas $80.95 \%, 66 \%, 52.38 \%, 16 \%, 33 \%, 83 \%$ and $83 \%$ were resistant to Cefotaxime $(30 \mu \mathrm{g})$, Amoxicillin $(25 \mu \mathrm{g})$, Gentamicin $(10 \mu \mathrm{g})$, Streptomycin $(10 \mu \mathrm{g})$, Nalidixic Acid $(30 \mu \mathrm{g})$, Norfloxacin $(10 \mu \mathrm{g})$ and Ampicillin $(10 \mu \mathrm{g})$ respectively. Almost $83.00 \%$ of Shigella $s p$. isolates were susceptible to Ceftazidime $(30 \mu \mathrm{g})$ and all the Shigella

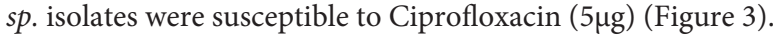




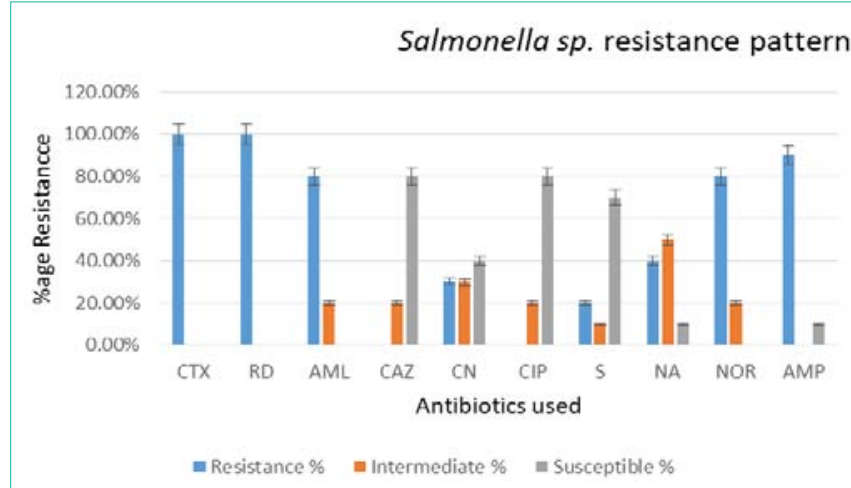

Figure 4: Chart presents resistance pattern of isolated Salmonella sp. strains against antibiotics used.

From all isolates of Salmonella sp. $100 \%$ were resistant to Cefotaxime $(30 \mu \mathrm{g})$ and Rifampicin $(5 \mu \mathrm{g})$. Moreover, $80.00 \%, 30.30 \%$, $20.00 \%, 40.00 \%, 80.90 \%$, and $90.32 \%$ were resistant to Amoxicillin $(25 \mu \mathrm{g})$, Gentamicin $(10 \mu \mathrm{g})$, Streptomycin $(10 \mu \mathrm{g})$, Nalidixic Acid $(30 \mu \mathrm{g})$, Norfloxacin $(10 \mu \mathrm{g})$ and Ampicillin $(10 \mu \mathrm{g})$ respectively. Whereas, $80 \%$ of the Salmonella sp. isolates were susceptible to

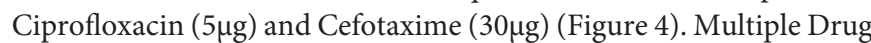
Resistance (MDR) was observed in Shigella sp. and Salmonella sp. isolates where, one Salmonella sp. isolate showed resistance to four antibiotics i.e. Cefotaxime, Rifampicin, Ampicillin and Norfloxacin, while $80 \%$ of the isolates exhibited multiple drug resistance to various antibiotics tested in the study.

\section{Discussion}

Enteric pathogens and their potential role in developing malnutrition and stunting have been the subject of interest in research in particular keeping in view prevailing environmental stress or sanitation \& hygiene conditions in Africa and Asia. Enteric pathogens introduced in earlier life may compromise the intestinal barrier, increase inflammation, lead to micronutrient deficiencies and chronic immune stimulation, which may increase the susceptibility to infections and impair growth [23]. Many pathogenic bacteria and viruses have been associated with stunted growth along with socioeconomic factor. However little is known about the factor that leads to stunting among children in the Pakistani pediatric population, therefore the main objective of this study was to determine the prevalence and antibiotics resistance pattern of Salmonella and Shigella sp. in stunted children under five years of age from the lower socioeconomic background of Pakistan. In the present study, the Salmonella sp. (10.5\%) was found to be predominantly associated with stunted children (having no sign and symptoms of diarrhoea), which higher than the earlier report from Pakistan (7.6\%) and Bangladesh (2\%) $[24,25]$. Pathogenic non-typhoid Salmonella sp. disrupts the commensal microbiota of the host and capable to colonize in the gut region [26]. Salmonellosis has been associated earlier with ProteinCalorie Malnutrition (PCM) which compromises mucosal epithelial barriers in the gastrointestinal tract. The loss of the protective blanket increases susceptibility to infection by pathogenic microbes [27].

Shigella sp. and toxigenic E. coli were found in asymptomatic, malnourished children as well as with poor sanitation and hygienic conditions $[28,29]$. The current study has shown a significant correlation between the presence of Shigella sp. with childhood malnutrition \& stunting. These findings can be interpreted by peculiar socio-cultural conditions, socio-economic parameters and general hygienic practices of the referred societies. In contrast to the earlier report, the higher number of samples were positive of shigella among stunted children i.e., 5\% [30] (Figure 5). According to this study, the resistance of Salmonella $s p$. was $80 \%$ to amoxicillin and 90 $\%$ to ampicillin, which is following the earlier reports from Karachi Pakistan [31,32]. The possible explanation could be due to the wide use of this drug in the country and frequent exposure of microbes to this antibiotic. However, the current study has shown the association of lower resistance of Salmonella sp. to gentamycin i.e. $30.30 \%$, this is in deviation from a study conducted in Al-Qasimi Hospital Sharjah, where Salmonella sp. have shown $100 \%$ sensitivity to gentamycin [33]. Moreover, this study has reported $80 \%$ susceptibility of Salmonella sp. isolated from stunted children to ceftazidime, which is following a

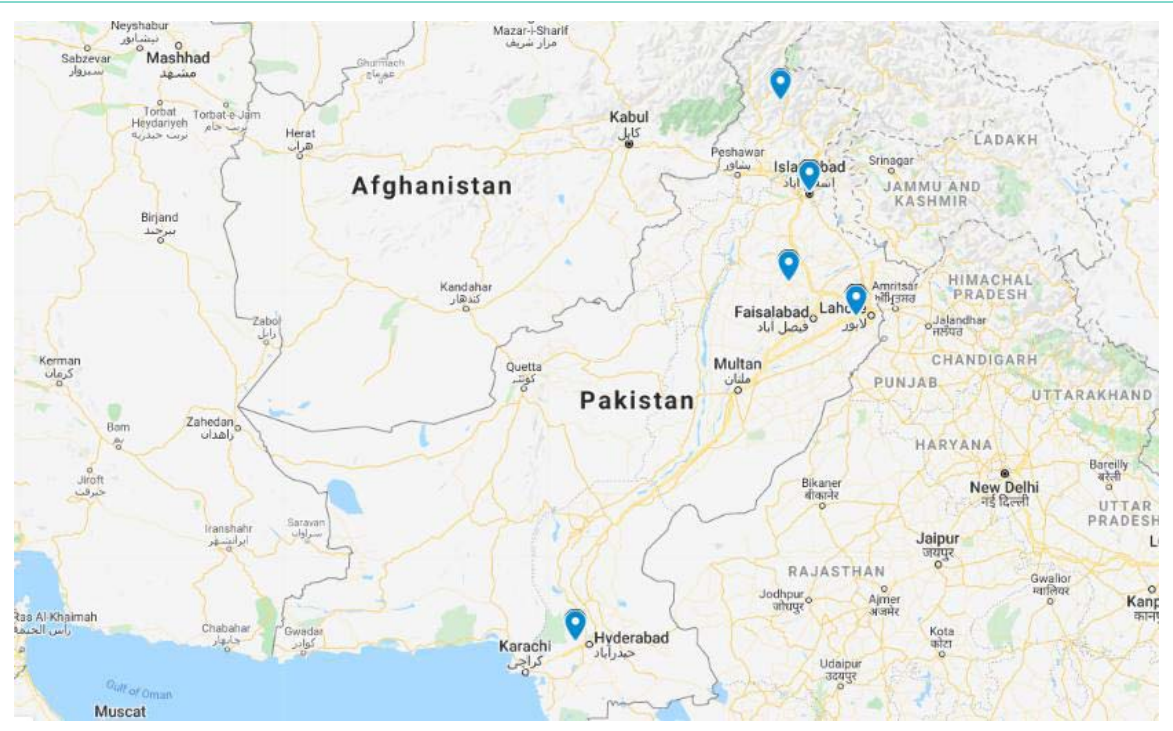

Figure 5: Map for Sample collection. 
previous study conducted in Lahore Pakistan, in which Salmonella sp. showed $85 \%$ sensitivity to ceftazidime [34]. In a study from India, less than $2 \%$ isolates of Salmonella sp. showed resistance to ciprofloxacin [35], whereas, in our study all the isolates of Salmonella sp. exhibited sensitivity towards ciprofloxacin.

A study conducted in Bangladesh has reported a rise in resistant strains of Shigella sp. towards ciprofloxacin from $0 \%$ in 2004 to $44 \%$ in 2010 [36], perversely in this study all the isolates of Shigella sp. have exhibited susceptibility towards ciprofloxacin. In Pakistan, nalidixic acid is an inexpensive drug and it is frequently administered to treat acute diarrhoea in children from poor resource settings. But the role of nalidixic acid as an empirical antibiotic for shigellosis has been compromised in our population, as $33 \%$ of Shigella sp. isolates were found to be resistant to nalidixic acid.

Poultry and livestock are a good source of protein in the form of eggs, meat and milk, however various studies on poultry and poultry feed have reported the increased incidence of multidrug-resistant Salmonella and Shigella in poultry feed [37], which can lead to possible vertical and horizontal transmission from an infected bird to the consumers $[38,39]$. Moreover, close association with the livestock and poor sanitation/hygiene may lead to frequent exposure to these enteropathogens. Safe livestock farming to prevent exposure to enteropathogens such as Salmonella and Shigella sp. will help children to grow to full potential by allowing their small intestine absorptive capacity to work to full potential.

\section{Conclusion}

It's an alarming situation that Pakistan is ranked top of the list, with $38 \%$ of children showing stunted growth. Poor socioeconomic condition, undernutrition, unhygienic food handling close association with animals and behavioural issues of communities and contaminated water are highly associated with enteric dysfunction or enteropathy leading to stunting in the pediatric population. Diarrheal pathogens Shigella $s p$. and Salmonella sp. (associated asymptomatically \& symptomatically) have been highly prevalent among children $<5$ years of age interfering in their linear growth. Although poultry and livestock are a good source of proteins, their proximity to children at the very early age of their life may expose them to enteropathogens which alters their gut functions and hence their nutrient absorption capacity. Moreover, frequent use of antibiotics as growth promoters in the poultry/livestock industry may engender the MDR of enterobacteria in human consumers. The study suggested the strong positive correlation of co-occurrence of multidrug-resistant Shigella $s p$. and Salmonella sp. with the gut of stunted children from a low socioeconomic group of Pakistan.

\section{Acknowledgement}

We gratefully acknowledge the Pediatrics departments of Mayo Hospital Lahore and PIMS Hospital Islamabad Pakistan for their support and collaboration in conducting sampling along with survey questionnaire.

\section{References}

1. Zangmo $U$, de Onis $M$, Dorji T. The nutritional status of children in Bhutan: results from the 2008 National nutrition survey and trends over time. BMC Pediatr, 2012; 12: 151.
2. Black RE, Victora CG, Walker SP, Bhutta ZA, Christian P, De Onis M, et al. Maternal and child undernutrition and overweight in low-income and middleincome countries. The Lancet, 2013; 382: 427-451.

3. Guerrant RL, Oriá RB, Moore SR, Oriá MO, Lima AA. Malnutrition as an enteric infectious disease with long-term effects on child development, Nutrition reviews, 2008; 66: 487-505.

4. Kossmann J, Nestel P, Herrera MG, Amin A, Fawzi WW. Undernutrition concerning childhood infections: a prospective study in Sudan. European journal of clinical nutrition, 2000; 54: 463

5. Collins S, Dent N, Binns $P$, Bahwere $P$, Sadler K, Hallam A. Management of severe acute malnutrition in children. The Lancet. 2006; 368: 1992-2000.

6. Tsedeke W, Tefera B, Debebe M. Prevalence of acute malnutrition (wasting) and associated factors among preschool children aged 36-60 months at Hawassa Zuria, South Ethiopia: a community-based cross-sectional study. Journal of Nutrition \& Food Sciences, 2016; 6: 466.

7. World Health Organization. analyser, WHO anthro survey. WHO anthro survey analyser. 2017.

8. World Health Organization, Unicef, World Health Organization, \& Unicef. WHO child growth standards and the identification of severe acute malnutrition in infants and children: a joint statement by the World Health Organization and the United Nations Children's Fund. 2009.

9. Walker SP, Wachs TD, Grantham-McGregor S, Black Nelson CA, Huffman $\mathrm{S}$., et al. Inequality in early childhood: risk and protective factors for early child development. The Lancet. 2011; 378: 1325-1338.

10. Kunwal N. One-third of the world's stunted children live in Pakistan. Daily times. 2019

11. WHO. Stunting policy brief (No. WHO/NMH/NHD/14.3). Global nutrition targets 2025. 2014

12. Zambrano LD, Levy K, Menezes NP, Freeman MC. Human diarrhoea infections associated with domestic animal husbandry: a systematic review and meta-analysis, Transactions of the Royal Society of Tropical Medicine and Hygiene. 2014; 108: 313-325.

13. Headey D, Nguyen P, Kim S, Rawat R, Ruel M, Menon P. Is exposure to animal faeces harmful to child nutrition and health outcomes? A multicountry observational analysis, The American journal of tropical medicine and hygiene. 2017; 96: 961-969.

14. George CM, Oldja L, Biswas S, Perin J, Lee GO, Kosek M, et al. Geophagy is associated with environmental enteropathy and stunting in children in rural Bangladesh. The American journal of tropical medicine and hygiene, 2015;. 92: 1117-1124.

15. Mbuya MN, Humphrey $\mathrm{JH}$. Preventing environmental enteric dysfunction through improved water, sanitation and hygiene: an opportunity for stunting reduction in developing countries, Maternal \& child nutrition. 2016;12: 106120.

16. Headey D, Nguyen P, Kim S, Rawat R, Ruel M, Menon P. Is exposure to animal faeces harmful to child nutrition and health outcomes? A multicountry observational analysis, The American journal of tropical medicine and hygiene, 2017; 96: 961-969.

17. Bern C, Martines J, De Zoysa I, Glass RI. The magnitude of the global problem of diarrhoeal disease: a ten-year update, Bulletin of the world health organization. 1992; 70: 705-714.

18. Tickell KD, Pavlinac PB, John-Stewart GC, Denno DM, Richardson BA, Naulikha JM, et al. Impact of childhood nutritional status on pathogen prevalence and severity of acute diarrhoea. The American journal of tropical medicine and hygiene. 2017; 97: 1337-1344.

19. Page AL, de Rekeneire N, Sayadi S, Aberrane S, Janssens AC. Rieux C, et al. Infections in children admitted with complicated severe acute malnutrition in Niger PloS one. 2013; 8: e68699.

20. Kader G, Nikkon F, Rashid MA, Yeasmin T. Antimicrobial activities of the rhizome extract of Zingiber zerumbet Linn, Asian Pacific Journal of Tropical Biomedicine. 2011; 1: 409-412. 
21. Vidal M, Kruger E, Durán C, Lagos R, Levine M, Prado V, et al. Single multiplex PCR assay to identify simultaneously the six categories of diarrheagenic Escherichia coli associated with enteric infections, Journal of clinical microbiology. 2005; 43: 5362-5365.

22. Ojha SC. A pentaplex PCR assay for the detection and differentiation of Shigella species. BioMed research international. 2013.

23. Saeki EK. Multiplex PCR (mPCR) for the Detection of Salmonella spp. and the Differentiation of the T Typhimurium and Enteritidis Serovars in Chicken Meat. Journal of food safety. 2013; 33: 25-29.

24. Ejaz MS. Clinical pattern of infections in malnourished children. Medical Channel. 2010; 16

25. Das SK. Aetiology of diarrhoea among severely malnourished infants and young children: Observation of urban-rural differences over one decade in Bangladesh. Food and Nutrition Sciences. 2010. 4: 233.

26. Barman MU. Enteric salmonellosis disrupts the microbial ecology of the murine gastrointestinal tract. Infection and immunity. 2008; 76: 907-915.

27. Rodríguez LC. Malnutrition and gastrointestinal and respiratory infections in children: a public health problem. International journal of environmental research and public health. 2011; 8: 1174-1205.

28. Vonaesch PT-V. Factors associated with stunting in healthy children aged 5 years and less living in Bangui (RCA). PloS one. 2017; 12: e0182363.

29. Ahmed KS. Aetiology of shigellosis in northern Pakistan. Journal of Health, Population and Nutrition. 2003; 32-39.

30. Vonaesch P, Tondeur L, Breurec S, Bata P, Nguyen LBL, Frank T, et al Factors associated with stunting in healthy children aged 5 years and less living in Bangui (RCA). PLoS One, 2017; 12
31. Saqib A. Culture and sensitivity of Salmonella species: analysis of a two-year data. J Pak Med Assoc. 2000, 50: 282-284

32. Aziz S. The emergence of Multi-Resistant Enteric Infection in a Paediatric Unit of Karachi, Pakistan. Age. 2018; 5: 2-84.

33. Damjanovic VFM. Antibiotic sensitivity of enteropathogenic bacteria isolated from patients in a Sharjah hospital. J Hyg. 1994; 92: 205-208.

34. Javaid HU. Changing patterns of antimicrobial susceptibility of salmonella Typhi at the children's hospital Lahore. Pakistan J Med Health Sci. 2012; 6: 201-204.

35. Bhattacharya SS. Occurrence \& antibiogram of Salmonella typhi \& S. paratyphi A isolated from Rourkela, Orissa. The Indian journal of medical research. 2011; 133: 431-433.

36. Azmi IJ. Fluoroquinolone resistance mechanisms of Shigella flexneri isolated in Bangladesh. PloS one, 2014; 9.

37. Ezekiel CN. Distribution, antibiogram and multidrug resistance in Enterobacteriaceae from commercial poultry feeds in Nigeria. Afr j Microbio Res. 2011 5: 294-301.

38. McEwen SA. Antimicrobial use and resistance in animals. Clinical infectious diseases. 2002; 34: S93-S106.

39. Young IR. Comparison of the prevalence of bacterial enteropathogens, potentially zoonotic bacteria and bacterial resistance to antimicrobials in organic and conventional poultry. Epidemiology \& Infection. 2009; 137: 12171232. 